\title{
Safety evaluation of chromium picolinate in experimental rats
}

\author{
Harish Kumar V. S. ${ }^{1 *}$, Sindhura Reddy Baleed ${ }^{2}$, Pradeep A. N. ${ }^{1}$, Kiran L. J. ${ }^{1}$, \\ Shivashankaramurthy K. G. ${ }^{1}$, Raghu Prasada M. S. ${ }^{1}$
}

\begin{abstract}
${ }^{1}$ Department of Pharmacology, S S Institute of Medical Sciences and Research Centre, Davangere, Karnataka, India
${ }^{2}$ Department of Emergency Medicine, JJMMC, Davangere, Karnataka, India
\end{abstract}

\author{
Received: 09 October 2019 \\ Revised: 12 December 2019 \\ Accepted: 13 December 2019 \\ *Correspondence: \\ Dr. Harish Kumar V. S., \\ Email: hkvspharma@gmail.com
}

Copyright: ( ) the author(s), publisher and licensee Medip Academy. This is an open-access article distributed under the terms of the Creative Commons Attribution Non-Commercial License, which permits unrestricted non-commercial use, distribution, and reproduction in any medium, provided the original work is properly cited.

\begin{abstract}
Background: Chromium, an essential trace mineral plays an important role in the metabolism of carbohydrate, fat and proteins. Chromium picolinate (Cr.Pic) is used in alternative medicine to treat chromium deficiency. Though Cr.Pic is increasingly used to treat diabetes and obesity, studies on its safety profile is limited.

Methods: Acute toxicity study was conducted by oral administration of Cr.Pic ( $2000 \mathrm{mg} / \mathrm{kg}$ body weight). The animals were maintained another 14 days with once a day observation. For sub-chronic studies, test groups were treated with Cr.Pic $10 \mathrm{mg} / \mathrm{kg} / \mathrm{day}$ for 90 days. Tests for hepatic and renal function were conducted. Effect of Cr.Pic on behavioural changes and motor co-ordination was done on every week. Histopathological studies were conducted on day 90 at the end of the experiment.

Results: Acute toxicity study of Cr.Pic showed no signs of toxicity and mortality. Absence of any behavioural alteration or mortality during the period of 14 days indicates that Cr.Pic has no latent effect. Similar results were obtained with sub-chronic studies suggesting safety of Cr.Pic. Cr.Pic treated groups showed no changes in learning and motor co-ordination compared to the untreated group. No gross histopathological changes were seen in any group indicating safety of Cr.Pic.

Conclusions: The present study conferred safety profile of Cr.Pic from normal results obtained in hepatic function, renal function, behavioural and histopathological studies, suggesting its safety.
\end{abstract}

Keywords: Acute toxicity, Chromium picolinate, Sub-chronic toxicity

\section{INTRODUCTION}

Chromium picolinate is a chemical compound derived from Chromium and picolinic acid. It has been used in alternative medicine to treat chromium deficiency, as an aid in controlling blood sugar in diabetics or prediabetics, to lower cholesterol and to promote weight loss. ${ }^{1}$ Chromium is an essential trace mineral that occurs naturally in small amounts in some foods, including brewer's yeast, lean meat, cheese, pork kidney and whole grain bread and cereals. It is poorly absorbed by the human body but is known to play an important role in the metabolism of carbohydrate, fat and protein. $^{2}$ Several pharmacological agents such as insulin-sensitizing agents may be used to reduce or control the body weight and obesity. One of such agents, chromium $(\mathrm{Cr})$ has been examined in some animal studies and clinical studies for its anti-obesity effects. ${ }^{3}$ Inadequate amounts of Chromium may result in improper functioning of the metabolic process and lead to a number of physiological disorders that increase risk for diabetes and cardiovascular diseases including elevated circulating insulin, glucose, triglycerides, total cholesterol, reduced HDL-cholesterol and impaired immune function. ${ }^{4,5}$ Dietary deficiency of chromium is believed to be positively 
associated with the risk of diabetes and its complications. ${ }^{6}$ In several animal and human studies, chromium complex of picolinic acid, the most popularly used dietary supplement, has been shown to modulate intracellular pathways of glucose metabolism and improve co-morbidities associated with insulin resistance. ${ }^{7,8}$

In a clinical trial, Martin and associates demonstrated an improvement in insulin sensitivity and glycated haemoglobin in subjects with type 2 diabetes who received chromium picolinate. ${ }^{9}$ Chromium picolinate is a stable compound for better absorption and it contains trivalent chromium which is chelated to three picolinic acid molecules. ${ }^{8}$ In recent years its usage has been increased for diabetes and obesity but there are not many studies on safety profile of Chromium Picolinate; thus this study was designed to evaluate the safety profile of Chromium picolinate (Cr.Pic) on rats by acute and sub chronic toxicity studies. The rats were grouped according to the diet that they were fed with and the treatment that they received. It is well documented that overeating and obesity can be produced in experimental animals like rats by offering them diets that are high in fat, sugar or both. The most pronounced effects are obtained when the animals are offered an assortment of tasty fat and sugar-rich foods marketed for human consumption, which is referred to as the cafeteria diet. ${ }^{10,11}$ This study is further implicated for evaluation of antihyperlipidaemic, anti-obesogenic and anti-atherosclerotic effects of Cr.Pic. So as to evaluate all these effects of Cr.Pic we fed few of the rats with cafeteria diet and grouped them accordingly. Our present study is carried out as a preliminary study to evaluate the safety profile of Cr.Pic even before getting into the study of its antihyperlipidaemic, anti-obesogenic and anti-atherosclerotic effects.

\section{METHODS}

\section{Grouping of wistar albino rats for acute toxicity study}

As per the IAEC guidelines acute toxicity study by oral route was carried out in two groups of wistar albino rats with 4 animals in each group ( 2 male and 2 female) to ascertain any mortality at the dose level of $2000 \mathrm{mg} / \mathrm{kg}$ body weight. Allocation of the groups for treatment was as follows:

Group I: rats served as control and were treated with $2 \mathrm{ml}$ of distilled water (D.W) and Group II: rats were given Cr.Pic $2000 \mathrm{mg} / \mathrm{kg}$ body weight in $2 \mathrm{ml}$ of D.W

Observations were made for any physical manifestation at 1, 2, 4 and 8 hours after oral administration. This included observation of skin and fur, eyes and mucous membranes, respiratory pattern, heart rate, autonomic and central nervous system manifestations and changes in behavior. Observation was made for any mortality during the 24 hour period following administration of the test materials. The animals were maintained for another 14 days with once a day observation. ${ }^{12}$

\section{Sub chronic study}

Sub chronic studies (90 days) were conducted to get data on safety profile and to find its toxic impact and pharmacological significance of biochemical as well as behavioural alterations consequent to repeated daily administration of the Cr.Pic. To correlate pharmacological significance of biochemical changes during daily treatment as well as to assess the toxic impact, the animals were monitored for behavioural parameters weekly, serum biochemical parameter analysis periodically at 30 days intervals and histopathological assessment done after sacrifice at the end of the experimental period.

\section{Grouping of rats for sub chronic study}

The rats were divided into 4 groups of six animals each for 90 days study. After 1 week of adaptation period treatment was started.

In Group 1, rats were fed normal-diet (ND) and treated with DW, in Group 2, rats were fed ND and treated with Cr.Pic. $10 \mathrm{mg} / \mathrm{kg} /$ day. Rats were fed cafeteria-diet (CD) and ND and treated with DW in Group 3 and rats were fed CD and ND and treated with Cr.Pic. $10 \mathrm{mg} / \mathrm{kg} /$ day in Group 4.

Cr.Pic solution was prepared fresh daily and the dose volume was adjusted to $1 \mathrm{ml} / \mathrm{day}$. Group 2 and 4 rats were treated with Cr.Pic every day. Group 3 and 4 received fixed quantity of $\mathrm{CD}$ as mentioned below as well as pellet-chow (ND) and water ad libitum for 90 consecutive days. Group 1 and 2 rats were fed only with pellet-chow and water ad libitum.

\section{Composition of $C D$}

The CD consisted of 3 diets, (a) condensed milk ( $8 \mathrm{gm})$ and bread (8 gm); (b) chocolate (3 gm) and biscuit (6 gm) with dried coconut (6 gm); (c) cheese ( $8 \mathrm{gm})$ and boiled potato (10 gm). The three diets were presented to the individual rats on day one, two and three, respectively, and then repeated in the same succession. ${ }^{13}$

\section{Sample collection and estimation of hepatic and renal function test}

During the treatment period, the animals were observed daily for toxic manifestations. Hepatic and renal function test were assessed on $0,30^{\text {th }}, 60^{\text {th }}$ and $90^{\text {th }}$ day of treatment. Blood was collected from the retro-orbital plexus of the rats after induction of mild anaesthesia with diethyl ether using micro capillary tubes for biochemical parameters. ${ }^{14}$ The tubes containing blood for biochemical analyses were kept in slant position for $2 \mathrm{hrs}$. The exuded serum was decanted and centrifuged at $1000 \mathrm{rpm}$ for 10 min. The clear supernatant serum was subjected to biochemical evaluations. 
The estimation of the levels of aspartate aminotransferase (AST), alanine aminotransferase (ALT), alkaline phosphatase (ALP), total protein, albumin, globulin, $\mathrm{A} / \mathrm{G}$ ratio, total bilirubin, direct bilirubin and indirect bilirubin as a part of hepatic function test and the levels of urea, creatinine and uric acid as a part of renal function test was done on the blood collected from all the rats once on $0,30^{\text {th }}, 60^{\text {th }}$ day of the study and then at term. The hepatic and renal functions were determined by using a commercially available reagent kit (Merck).

\section{Behavioural study}

All the rats were initially trained in the below instruments and thereafter once every week recordings were taken after the treatment to see the influence of Cr.Pic on behavioural changes and motor coordination.

\section{Maze}

The apparatus consists of two chambers A and B, separated by a maze. Rat was placed in chamber A and allowed to explore the maze and the reward (pellet-chow) was provided at chamber $\mathrm{B}$. The time taken by the rats to traverse the maze and to reach the food compartment was recorded and considered as the criteria for successful learning and memory activity. ${ }^{15}$

\section{Actophotometer}

It operates on photoelectric cells which are connected in circuit with a counter. The locomotor activity of rat is recorded when the beam of light falling on the photo-cell is cut off by it. ${ }^{16}$

\section{Rotarod}

This test was done to evaluate motor deficits in rats. Rationale of it is to make the animal to maintain itself on a rotating rod, the time for which is recorded in seconds and the animal having defective motor coordination drops from the rod. ${ }^{17}$

\section{Histopathological study}

At the end of the experiment (90 days), the animals were sacrificed with an overdose of ether. Visceral organs like brain, heart, lung, liver, kidney and spleen were removed. The tissues initially fixed in formalin were subjected to dehydration and then blocked in paraffin wax, which were then sectioned into ribbon and fixed on glass slides. Tissue sections stained with haematoxylin and eosin were examined by light microscopy for histopathological evaluations. $^{18}$

\section{Assessment of the results}

Within the group comparison was made between initial (day ' 0 ') reading and readings recorded at different intervals (durations) in the same animal to overcome the disparity in initial values between the different groups. In between group comparison was made, all the groups were compared with group 1.

\section{Statistical analysis}

For statistical significance $\mathrm{p}<0.05$ was kept as the minimum requirement.

\section{RESULTS}

\section{Acute toxicity study}

There was no mortality and no signs of toxicity up to 2000 $\mathrm{mg} / \mathrm{kg}$ body weight and hence it was considered to be safe.

\section{Sub chronic study}

Behavioral study

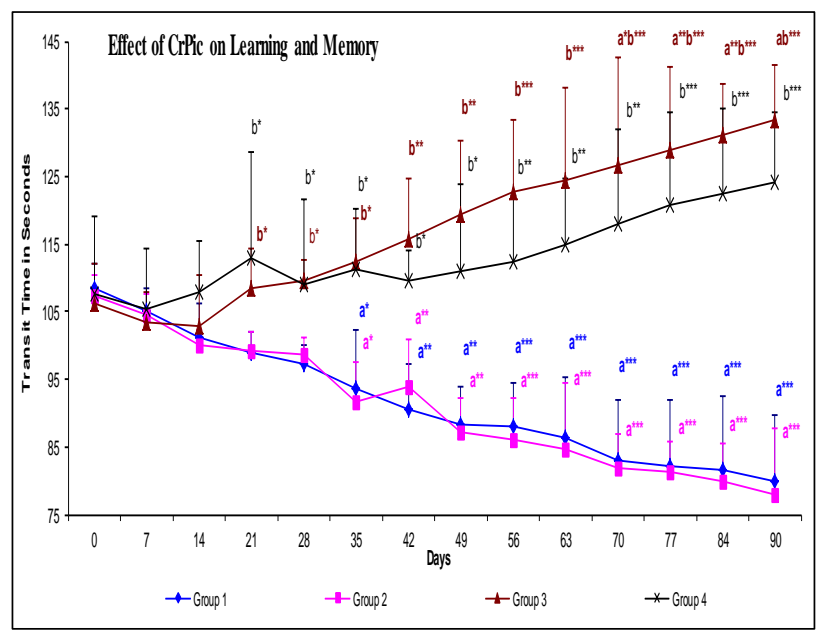

Figure 1: Effect of CrPic on learning and memory by maze in wistar albino rats fed with CD (Transit time in seconds).

Group 1: Normal Diet (ND), Group 2: ND + Cr Pic 10 mg/Kg, Group 3: Cafeteria Diet $(\mathrm{CD})+\mathrm{ND}$, Group 4: $\mathrm{CD}+\mathrm{ND}+\mathrm{Cr}$ Pic $10 \mathrm{mg} / \mathrm{Kg}, \mathrm{n}=6$. Mean \pm S.D. a - compared with day ' 0 ', $\mathrm{b}$ compared with group ' 1 '. ${ }^{*} \mathrm{p}<0.05, * * \mathrm{p}<0.01, * * * \mathrm{p}<0.001$.

Effect of CrPic on learning and memory by maze in wistar albino rats fed with $C D$ (transit time in seconds).

\section{Within group comparison}

Significant decrease in transit time was noticed in group 1 and 2 from day 35, whereas significant increase in transit time was seen in group 3 from day 70 when compared to their initial level (day 0).

\section{In between group comparison}

Significant increase in the transit time was observed in group 3 and 4 after 21 days of the study when compared to group 1 on their respective days. 


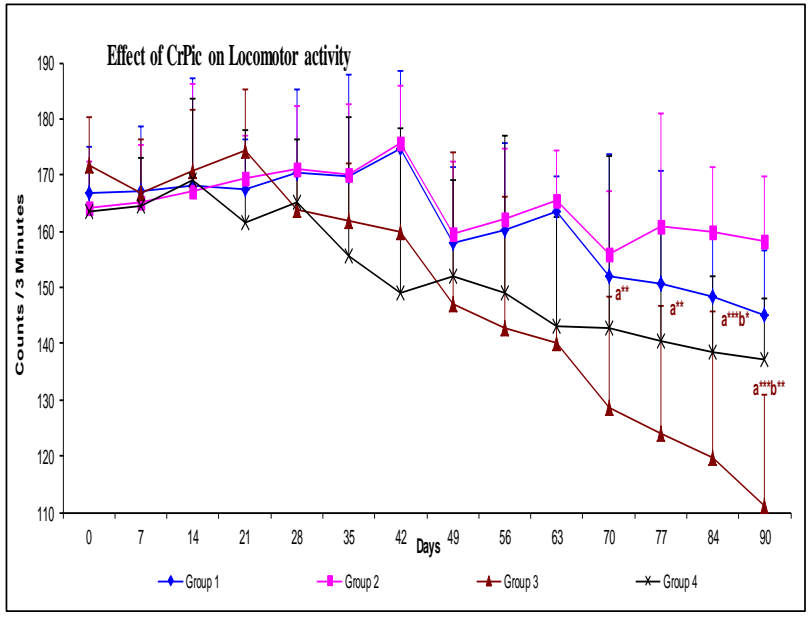

Figure 2: Effect of CrPic on locomotor activity by actophotometer in wistar albino rats fed with CD (counts/3 minutes).

Group 1: Normal diet (ND), Group 2: ND + Cr Pic $10 \mathrm{mg} / \mathrm{Kg}$, Group 3: Cafeteria Diet $(C D)+N D$, Group 4: $C D+N D+C r$ Pic $10 \mathrm{mg} / \mathrm{Kg}, \mathrm{n}=6$. Mean \pm S.D. a - compared with day ' 0 ', $\mathrm{b}$ compared with group ' 1 '. ${ }^{*} \mathrm{p}<0.05,{ }^{* *} \mathrm{p}<0.01,{ }^{* * *} \mathrm{p}<0.001$.

Effect of CrPic on locomotor activity by actophotometer in wistar albino rats fed with $C D$ (counts/3 minutes).

\section{Within group comparison}

Locomotor activity of the rats was found to be unaffected in all the groups except group 3, where a significant decrease in locomotor activity was noticed from $70^{\text {th }}$ day of the treatment.

\section{In between group comparison}

There was no statistical significance in any of the groups when compared to the control group at any time of the study whereas a significant decrease in locomotor activity was seen in group 3 on day $84 \& 90$ when compared to group 1 on their respective days.

\section{Effect of CrPic on motor coordination by rotarod in Wistar albino rats fed with CD (seconds).}

\section{Within group comparison}

Animals of the control as well as the test groups were able to maintain on the rotarod. There was no significant deficit in motor coordination seen in the groups except group 3, where a significant decrease in time was observed from day 63 compared to their initial level (day 0).

\section{In between group comparison}

There was no significant deficit in any of the groups when compared to the control group at any time of the study except group 3, where a significant decrease in motor coordination was noticed from day 49 when compared to group 1 on their respective days.

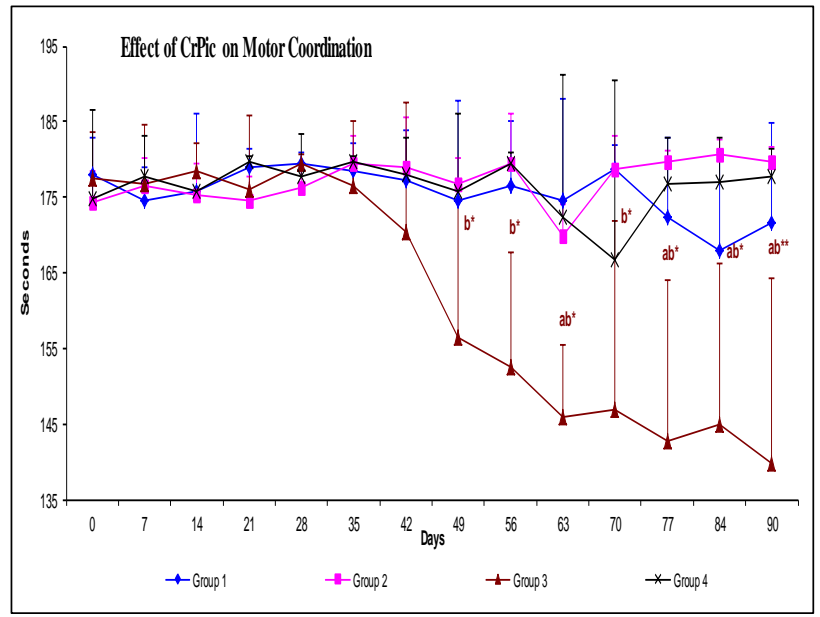

Figure 3: Effect of CrPic on motor coordination by rotarod in wistar albino rats fed with $\mathrm{CD}$ (seconds). Group 1: Normal Diet (ND), Group 2: ND + Cr Pic 10mg/Kg, Group 3: Cafeteria Diet (CD) + ND, Group 4: CD + ND + Cr Pic $10 \mathrm{mg} / \mathrm{Kg}, \mathrm{n}=6$. Mean \pm S.D. a - compared with day ' 0 ', b compared with group ' 1 '. * $\mathrm{p}<0.05,{ }^{* *} \mathrm{p}<0.01, * * * \mathrm{p}<0.001$.

\section{Effect of Cr.Pic on liver function test in ND fed rats and CD fed rats.}

\section{Within group comparison}

There was no significant change noticed in serum AST, albumin, total bilirubin, direct bilirubin and indirect bilirubin levels recorded at various intervals during the experimental period in all groups compared to their initial level (day 0).

Significant increase in serum ALT level was seen in group 4 on day 90. ALP level was increased in group 3 on day 60, 90 and in group 4 on day $90, \mathrm{~A} / \mathrm{G}$ ratio in group 3 was high on day 90 when compared to their initial level.

There was significant decrease in serum total protein and globulin in group 3 on day 90 compared to their initial level, this decrease in serum total protein and globulin was not seen with $\mathrm{Cr}$.Pic treated group.

\section{In between group comparison}

Compared with the group 1 (control), serum AST levels were significantly increased on day 30 and 60 in group 3 . The serum ALT levels were significantly decreased in group 3 on day 0,30 and 60 compared to the control group. Treatment with Cr.Pic in group 4 prevented this change in the serum levels of AST and ALT.

ALP levels in group 4 showed a significant increase on day 0, 30, 60 and 90 compared to control group. There was a significant decrease in serum Total Protein in group 3 on day 60 and 90 when compared to group 1, whereas treatment with Cr.Pic in group 4 prevented this decrease in total protein. A/G ratio was significantly increased in group 3 on day 90 compared to group 1 , whereas treatment with $\mathrm{Cr}$.Pic in group 4 prevented this increase in $\mathrm{A} / \mathrm{G}$ ratio. 
The other parameters in liver function test like albumin, globulin, total bilirubin, direct bilirubin and indirect bilirubin were all within normal limits in all the groups and there was no significant difference when compared to control group.
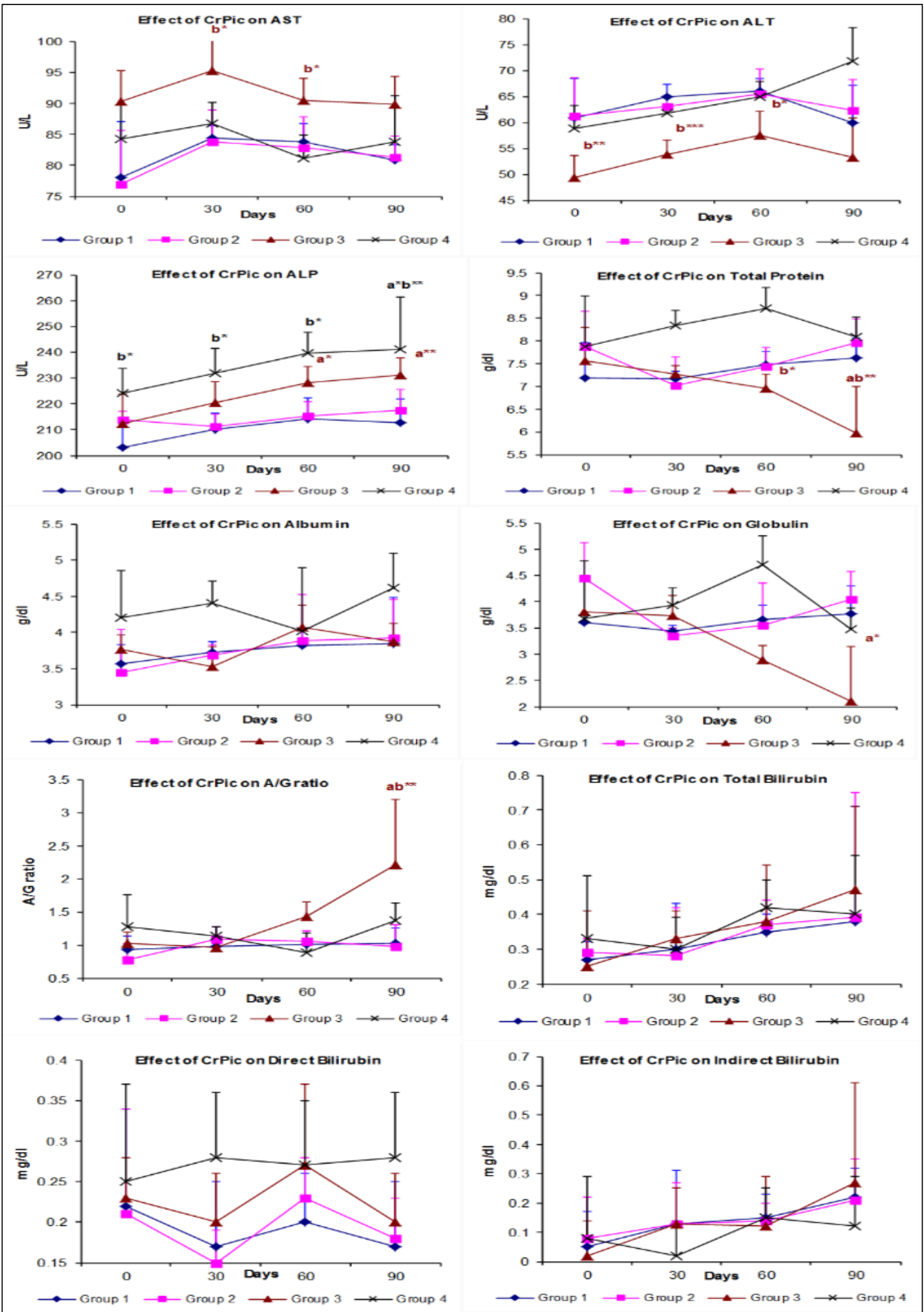

Figure 4: Effect of Cr.Pic on liver function test in ND fed rats and CD fed rats.

Group 1: Normal Diet (ND), Group 2: ND + Cr Pic $10 \mathrm{mg} / \mathrm{Kg}$, Group 3: Cafeteria Diet (CD) + ND, Group 4: CD + ND + Cr Pic 10 $\mathrm{mg} / \mathrm{Kg}, \mathrm{n}=6$. Mean \pm S.D. a - compared with day ' 0 ', $\mathrm{b}$ - compared with group ' 1 '. ${ }^{*} \mathrm{p}<0.05,{ }^{* *} \mathrm{p}<0.01,{ }^{* * *} \mathrm{p}<0.001$. 


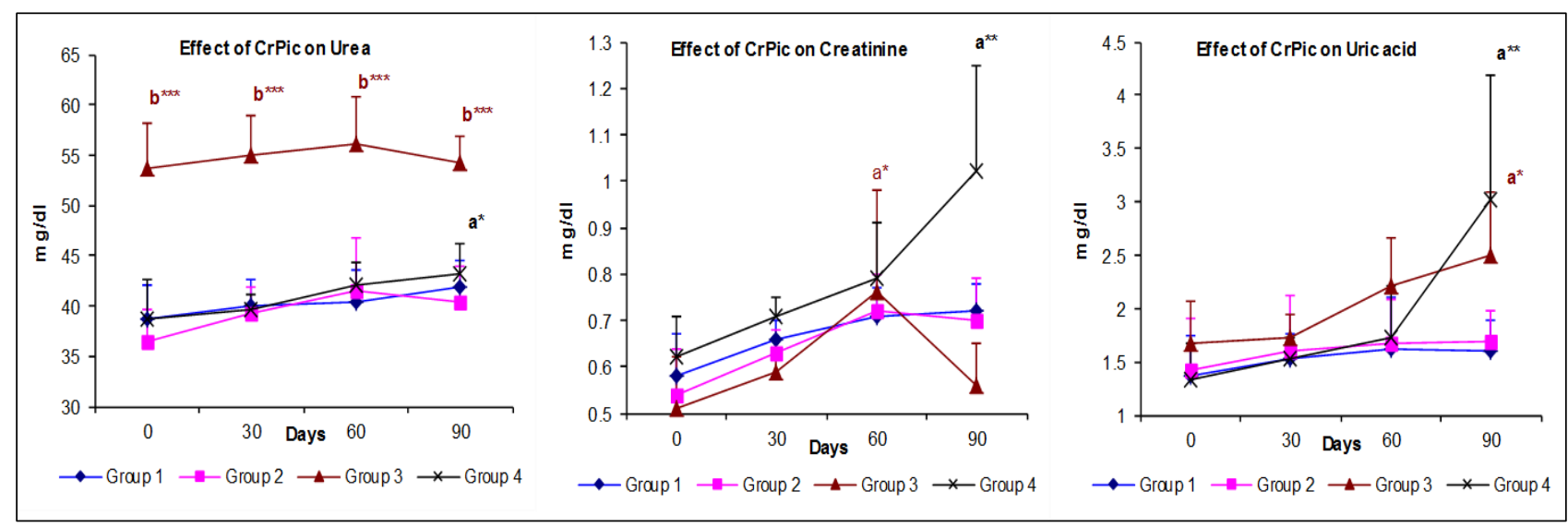

Figure 5: Effect of Cr.Pic on renal function test in ND fed rats and CD fed rats.

Group 1: Normal Diet (ND), Group 2: ND + Cr Pic $10 \mathrm{mg} / \mathrm{Kg}$, Group 3: Cafeteria Diet (CD) + ND, Group 4: CD + ND + Cr Pic $10 \mathrm{mg} / \mathrm{Kg}, \mathrm{n}=6$. Mean \pm S.D. a - compared with day ' 0 ', $\mathrm{b}$ - compared with group ' 1 '. ${ }^{*} \mathrm{p}<0.05, * * \mathrm{p}<0.01,{ }^{* * *} \mathrm{p}<0.001$.

Within group comparison: There was no significant change in serum urea, creatinine and uric acid levels recorded at various intervals during the experimental period in any of the groups except group 4 on day 90 compared to their initial urea level (day 0). Increased serum creatinine level was seen in group 3 on day 60 and group 4 on day 90 compared to their initial level. Significant increase in uric acid level was observed in group 3 and 4 on day 90 compared to their initial level.

In between group comparison: Compared to the control group, a significant increase in the levels of urea has been observed on all the respective days of study in group 3, while in group 2 and 4 there is no much statistical difference in comparison to the control. There was no significant change in creatinine and uric acid in any of the groups compared to control (group 1) throughout the study.

\section{Histopathological study (Figure 6)}

At the end of the experiment, control rats as well as animals treated with Cr.Pic showed no abnormal postmortem findings. Treatment with Cr.Pic has not caused any morphological alteration in liver, heart, kidney, brain, lung and spleen in any of the rats. The histopathological assessment has not shown any toxic effect in the essential tissues. In all these tissues the normal architecture has not been disturbed and no specific lesion was seen. Normal architecture of hepatic lobules, hepatocytes, portal triads, sinusoids was all well preserved in rats treated with Cr.Pic. There was no histopathological change observed in sections of liver in any of the groups except for mild steatosis in group 3 rats. Sections of heart from control as well as Cr.Pic treated rats showed no adverse lesions. Sections of kidney showed normal architecture of glomeruli and tubules in all the rats. No specific lesion was seen in sections of brain, lung and spleen in any of the groups.

Liver (40x) $(\mathrm{G}=$ Group $)$

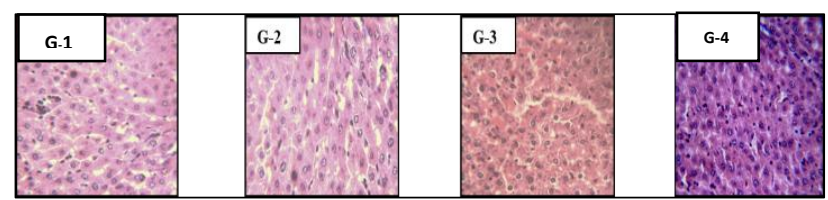

\section{Kidney (40x)}

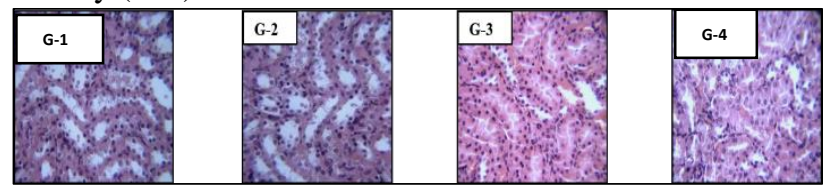

Lung (40x)

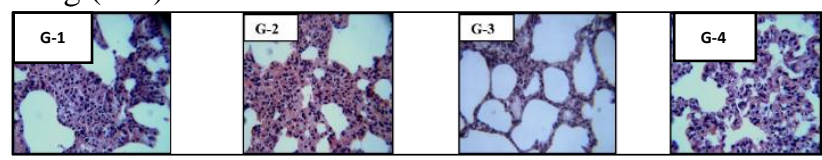

Heart (40x)

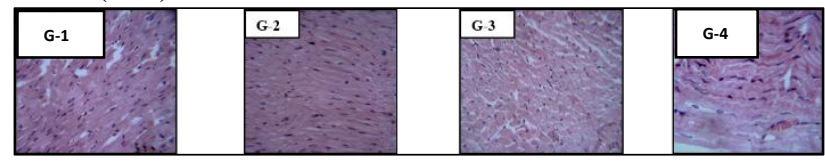

Brain (40x)

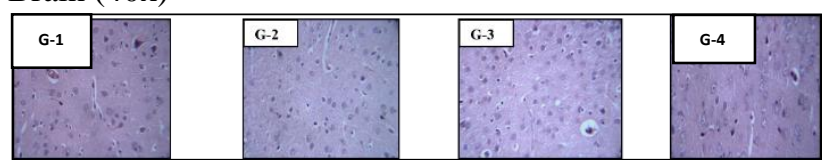

Spleen (40x)

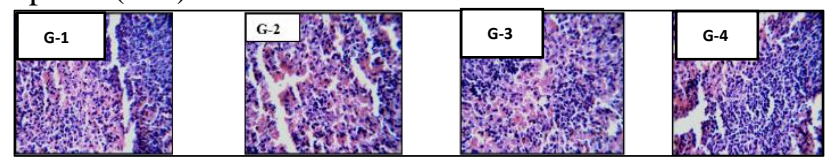

Figure 6: Histopathological study.

\section{DISCUSSION}

Acute toxicity study was conducted by oral administration of Chromium Picolinate ( $2000 \mathrm{mg} / \mathrm{kg}$ body weight). Cr.Pic was non-lethal as no mortality was seen. Absence of any behavioral alteration or mortality during the post-observation period of 14 days indicates that administration of a single 
dose of Cr.Pic has no latent effect. Absence of mortality due to daily administration of Cr.Pic (10 mg/kg body weight) in the sub chronic study is also indicative of the safety during long-term administration.

Administration of Cr.Pic (10 mg/kg body weight) for 90 days (sub chronic study) has not caused any gross variation in the normal ranges of the serum biochemical parameters studied, ruling out the possibility of any toxic effect and thus can be considered as safe. Absence of any pathological change investigated by histopathological studies is furthermore supportive of this.

Toxicity studies in animals are commonly used to assess potential health risk in humans caused by intrinsic adverse effects of chemical compounds. These adverse effects may manifest significant alterations in the levels of biomolecules such as enzymes and metabolic products, normal functioning and histomorphology of the organs. ${ }^{19}$

Administration of Cr.Pic for 90 days seems to have no injurious effect on the neuronal functions. Deficits in neuronal function can lead to alterations in cognitive and motor behaviors. ${ }^{20}$ In animal models, cognitive function is usually measured in a maze and motor function is assessed by the ability of a rodent to maintain itself on a slowly rotating rod.

The study on the behaviour of rats here showed that the treatment with Cr.Pic produced no defective motor coordination or any other motor or learning deficits in any of the groups. While assessing the learning and memory of rats by maze, a statistically significant increase in the transit time was seen in group $3 \mathrm{CD}$ fed rats from the 70th day of study. This could be attributed to the rats' lack of interest on the reward (pellet chow) as they were fed with the much more tastier cafeteria diet every day and this would have happened as a part of their learning and memory skills in the due course of 70 days time. However in group 1 and $\mathrm{Cr}$.Pic treated group 2 rats showed a decrease in transit time through the maze. This could be because they were not fed with Cafeteria Diet (CD) and hence have shown interest in the reward (pellet chow). Treatment with Cr.Pic in CD fed rats compared to their initial transit time showed no significant change. The transit time through maze is found to be unaffected during treatment of Cr.Pic for 90 days. This is an indication of the absence of any impairment of working memory which describes the ability of the subject to hold specific information, like places previously visited, in memory. ${ }^{21}$

Treatment with the Cr.Pic for 90 days has not altered the motor activity as the actophotometer counts are more or less similar in group 1 and Cr.Pic treated groups. However, in group 3 animals there was a decrease in the motor activity in last few weeks of experimental period. This could be because of increase in body weight of the rats as they were fed with high fat diet (CD).
Deficits in motor function can result in decrease in balance, muscle strength and coordination. The ability of an animal to maintain itself on a rotating rod requires the components of learning and motor coordination. Motor coordination has been assessed in the present study by rota-rod performance. Motor coordination has not been hindered by Cr.Pic during 90 days of exposure. From the result of the rota-rod performance, it could be seen that treatment with $\mathrm{Cr}$.Pic has not caused any deficit in learning and motor coordination as the animals belonging to the group 1, 2 and 4 were able to maintain on the rotarod for the trained duration of 180 seconds. A mild declining trend in the performance in last few weeks of experimental period was seen in $\mathrm{CD}$ fed untreated rats (group 3) and this could be because of increase in body weight of the rats.

Repeated administration of Cr.Pic (10 mg/kg/day) for 90 days has not affected the learning \& memory, loco motor activity and motor coordination of the experimental rats as per the results observed in the study. Thus there were no alterations in the behavior, suggesting its safety.

Liver is the major organ responsible for metabolism of toxic substances that enter the body and the functions of the liver can be detrimentally altered by liver injury resulting from acute or chronic exposure to toxicants or by situations affecting both B-oxidation and the respiratory chain enzymes. Further, hepatotoxicity has been viewed as liver injury associated with impaired liver function caused by exposure to drug or other noninfectious agents or herbs. Serum enzyme activities are used as indicators of chemical-induced liver damage. The study on the activities of different biomarkers such as AST, ALT, ALP, Bilirubin and the concentration of Total Protein have been found to be of great value in the assessment of clinical and experimental liver damage. Membrane damage releases the enzymes into circulation and therefore they can be measured in serum. ${ }^{22}$ Evaluation of serum total proteins, albumin and globulin are good criteria for assessing the secretory ability/functional capacity of the liver. ${ }^{19}$ Albumin levels are usually reduced in chronic liver diseases, congestive heart failure and nephritis. Increase in the serum bilirubin may arise from excessive haemolysis, cytotoxicity to the liver or from obstruction in bile ducts resulting in cholestasis. ${ }^{23}$ Total Bilirubin concentration indicates the functional transport capacity as well as conjugating ability of liver. ${ }^{24}$ In the present study significant changes are observed in AST, ALT, ALP, Total Protein, Globulin, A/G ratio in CD fed group 3 and group 4 (treated with Cr.Pic) but this changes were not seen in group 2 treated with $\mathrm{Cr}$.Pic which were fed with ND alone. This change in serum biochemical parameters in group 3 and 4 is not accompanied by any structural damage as revealed by the histology of liver in animals treated with $\mathrm{Cr}$.Pic fed with $\mathrm{CD}$, thus except for some functional alteration which could be due to cafeteria diet, there was no structural alteration in liver indicating absence of any permanent damage. 
Kidney is a susceptible organ to toxic injuries by drugs and toxins, because of a high blood supply and the presence of cellular transport systems that cause accumulation of these compounds within the nephron epithelial cells. Glomerular, tubular and interstitial cells frequently encounter significant concentrations of medications and their metabolites, which can induce changes in kidney function and structure. Renal toxicity can be a result of hemodynamic changes, direct injury to cells and tissue, inflammatory tissue injury and/or obstruction of renal excretion. ${ }^{25}$ As a measure of renal function status, serum urea, creatinine and uric acid are often regarded as reliable markers. In the present study, no significant alteration in serum urea, creatinine and uric acid was seen during exposure to Cr.Pic treatment for longer duration in ND fed rats. Whereas increase in serum urea, creatinine and uric acid level was seen in CD fed rats, group 3 and 4. This increase in the level of serum urea, creatinine and uric acid could be attributed to cafeteria diet. However no toxic impact has been observed in histology of the experimental rat's kidney. Thus there was no pathological change in renal sections of Cr.Pic treated groups and the sections were similar to that of the control group. This indicates the absence of any renal damage.

Histopathological examination revealed mild hepatic steatosis induced by cholesterol rich high fat diet, CD. These effects were not observed when the rats were given cholesterol rich $\mathrm{CD}$ along with $\mathrm{Cr}$.Pic. There were no pathological changes in the liver, kidney, heart, lung, spleen and brain of Cr.Pic treated groups. An oral administration of Cr.Pic ( $10 \mathrm{mg} / \mathrm{kg} /$ day) for 90 days has not caused any structural alteration in liver, kidney, heart, lung, spleen and brain. In all these tissues the normal architecture has not been disturbed and no specific lesion was seen except for a mild congestion in some of the animals. These observations assessed in conjunction with the results of the biochemical parameters, indicates that repeated administration of the Cr.Pic (10 mg/kg/day) for 90 days has not produced toxic impact on essential organs. Hence, the possibility of any toxic effect is ruled out and thus it can be considered to be safe.

\section{CONCLUSION}

This study conferred reasonable safety profile of Cr.Pic (10 $\mathrm{mg} / \mathrm{kg} / \mathrm{day}$ ) from normal results obtained in hepatic function, renal function, behavioral, learning and motor co-ordination and histopathological studies, suggesting its safety in rats. On further extensive clinical studies, Cr.Pic can be considered as safe.

Funding: No funding sources

Conflict of interest: None declared

Ethical approval: The study was approved by the Institutional Ethics Committee

\section{REFERENCES}

1. Preuss HG, Echard B, Perricone NV, Bagchi D, Yasmin T, Stohs SJ. Comparing metabolic effects of six different commercial trivalent chromium compounds. J Inorganic Biochem. 2008;102(11):1986-90.

2. Chromium Picolinate Demonstrates Diabetes Benefits. Availabe at: http://www.rejuvenationscience.com/n_chromium-picolinate_diabetes $2 . h t m l$. Accessed on 24 October 2017.

3. Pittler $\mathrm{MH}$, Stevinson C, Ernst E. Chromium picolinate for reducing body weight: Meta-analysis of randomized trials. Int J Obesity. 2003;27:522-9.

4. Mertz W. Chromium in human nutrition: a review. J Nutr. 1993;123:626-33.

5. Anderson RA. Chromium, glucose tolerance, diabetes. Biol Trace Elem Res. 1992;32:19-24.

6. Jeejeebhoy KN, Chu RC, Marliss EB, Greenberg GR, Bruce-Robertson A. Chromium deficiency, glucose intolerance, and neuropathy reversed by chromium supplementation, in a patient receiving long-term total parenteral nutrition. Am J Clin Nutr. 1977;30:531-8.

7. Anderson RA, Cheng N, Bryden NA, Polansky MM, Cheng N, Chi J, Feng J. Elevated intakes of supplemental chromium improve glucose and insulin variables in individuals with type 2 diabetes. Diabetes. 1997;46(11):1786-91.

8. Mozaffari MS, AbdelSayed R, Liu JY, Wimborne H, El-Remessy A, El-Marakby A. Effects of chromium picolinate on glycemic control and kidney of the obese Zucker rat. Nutrit Metab. 2009;6(1):51.

9. Martin J, Wang ZQ, Zhang XH, Wachtel D, Volaufova J, Matthews DE, et al. Chromium picolinate supplementation attenuates body weight gain and increases insulin sensitivity in subjects with type 2 diabetes. Diab Care. 2006;29(8):1826-32.

10. Sclafani A. Dietary obesity. In: Stunkard AJ, Wadden TA, editors. Obesity: theory and therapy. New York: Raven Press; 1993; 125-136.

11. Warwick ZS, Schiffman SS. Role of dietary fat in caloric intake and weight gain. Neurosci Biobehav Rev 1992;16:585-96.

12. Halim1 SZ, Abdullah NR, Afzan, Rashid A, Jantan, Ismail. J Med Plants Res. 2011;5:1867-72.

13. Kumar S, Alagawadi KR, Rao MR. Effect of Argyreia speciosa root extract on cafeteria dietinduced obesity in rats. Ind $J$ Pharmacol. 2011;43(2):163.

14. Harish Kumar VS, Vinutha MB, Pradeep AN, Sathisha Aithal SR, Umakant NP. Bromocriptine, a Dopamine (d2) Receptor Agonist, Used Alone and in Combination with Glipizide in Sub-Therapeutic Doses to Ameliorate Hyperglycaemia. J Clin Diag Res: JCDR. 2013;7(9):1904.

15. Jarvik ME. Techniques for evaluating the effects of drugs on memory. In: Animal and Clinical Pharmacological Techniques in Drug Evaluation. Eds. Nodine, J.H. and Seigler, P.E., Year Book Medical Publishers Inc. Chicago. 1964: 339-347.

16. Robert KS, Lim MB. Animal Techniques for evaluating Hypnotics. Animal and Clinical Pharmacological Techniques in Drug Evaluation. 
Eds. Nodine JH and Seigler PE. Chicago: Year Book Medical Publishers Inc; 1964: 291-297.

17. Jacobsen E. Tranquillisers and Sedatives. IN Evaluation of Drugs Activities: Pharmacometrics. Eds. Laurance DR, Bacharach. A.L, London: Academic Press; 1964, 1:215-237.

18. Bancroft JD, Cook HC. Edinburgh: Churchill Livingstone, Manual of Histological Techniques. 1984: 201-202.

19. Ashafa AO, Orekoya LO, Yakubu MT. Toxicity profile of ethanolic extract of Azadirachta indica stem bark in male Wistar rats. Asian Pacific J Trop Biomed. 2012;2(10):811-7.

20. Joseph JA, Bartus RT, Clody D, Morgan D, Finch C, Beer B, Sesack S. Psychomotor performance in the senescent rodent: reduction of deficits via striatal dopamine receptor up-regulation. Neurobiol Aging 1983;4(4):313-9.

21. Luine VN, Richards ST, Wu VY, Beck KD. Estradiol enhances learning and memory in a spatial memory task and effects levels of monoaminergic neurotransmitters. Horm Behav. 1998;34(2):149-62.
22. Sakuntala Devi PR, Adilaxmamma K, Rao GS, Raj MA. Safety evaluation of alcoholic extract of Boswellia ovalifoliolata stem-bark in rats. Toxicol Int. 2012;19:115-20.

23. Okokon JE, Nwafor PA, Ekpo MD. Subchronic toxicity studies of the ethanolic root extract of Croton zambesicus. Pakistan J Pharma Sci. 2010;23(2).

24. Dawane JS, Borole KD, Pandit VA, Dhrubajyoti D, Sahane SS. Karandikar MN Evaluation of Nephro. Hepato and Gastro toxic potential of aqueous extract of Dashamula. Int $\mathrm{J}$ Res Pharm Biomed Sci. 2012;3:13-9.

25. Kumar A, Kumari SN, D'Souza P, Bhargavan D. Evaluation of renal protective activity of Adhatoda zeylanica (medic) leaves extract in wistar rats. Nitte Uni J Health Sci. 2013;3(4):45.

Cite this article as: Kumar HVS, Baleed SR, Pradeep AN, Kiran LJ, Shivashankaramurthy KG, Prasada R. "Safety evaluation of chromium picolinate in experimental rats". Int J Basic Clin Pharmacol 2020;9:113-21. 